\title{
Evolution of very small particles in the southern part of Orion B observed by ISOCAM
}

\author{
A. Abergel ${ }^{1}$, J. P. Bernard ${ }^{1}$, F. Boulanger ${ }^{1}$, D. Cesarsky ${ }^{1,12}$, E. Falgarone ${ }^{2}$, A. Jones ${ }^{1}$, \\ M.-A. Miville-Deschenes ${ }^{1,2}$, M. Perault ${ }^{2}$, J.-L. Puget ${ }^{1}$, M. Huldtgren ${ }^{3}$, A. A. Kaas ${ }^{3}$, L. Nordh ${ }^{3}$, \\ G. Olofsson ${ }^{3}$, P. André ${ }^{4}$, S. Bontemps ${ }^{10}$, M. M. Casali ${ }^{11}$, C. J. Cesarsky ${ }^{4}$, M. E. Copet ${ }^{6}$, J. Davies ${ }^{7}$, \\ T. Montmerle ${ }^{4}$, P. Persi ${ }^{8}$, and F. Sibille ${ }^{9}$ \\ 1 Institut d'Astrophysique Spatiale, Université Paris-Sud, Bât. 121, 91405 Orsay Cedex, France \\ 2 Radioastronomie Millimétrique, École Normale Supérieure, 24 rue Lhomond, 75005 Paris, France \\ 3 Stockholm Observatory, 13336 Saltsjöbaden, Sweden \\ 4 Service d'Astrophysique, Centre d'Études de Saclay, 91191 Gif-Sur-Yvette Cedex, France \\ 5 ISO, Villafranca del Castillo, ESA Satellite tracking station, POB 50727, 28080 Madrid, Spain \\ 6 DESPA, Observatoire de Paris-Meudon, 5 place Jules Janssen, 92195 Meudon Cedex, France \\ 7 Joint Astronomy Center, 660 N. A'Ohoku Place, University Park, Hilo, HI 96720, USA \\ 8 Istituto Astrofisica Spaziale, CNR, Area di Ricerca Tor Vergata, Roma, Italy \\ 9 Observatoire de Lyon, 69230 Saint Genis Laval, France \\ 10 Observatoire de Bordeaux, BP 89, 33270 Floirac, France \\ 11 Royal Observatory, Blackford Hill, Edinburgh, UK \\ 12 Max Planck Institut fur extraterrestrische Physik, 85741 Garching, Germany
}

Received 30 July 2001 / Accepted 28 February 2002

\begin{abstract}
We present ISOCAM observations $(5-18 \mu \mathrm{m})$ of the southern part of Orion B, including the reflection nebula NGC 2023 and the Horsehead nebula illuminated by the B star HD 37903 and the O star $\sigma$ Orionis, respectively. Due to the limited radiation field, the emission in these wavelengths is due to very small particles which are heated each time they absorb a UV photon. A filamentary structure is detected at small angular scales (down to the angular resolution of $6^{\prime \prime}$ ) on top of a smoother background. The particular case of the Horsehead nebula suggests that the filaments in general result from the illuminated surfaces of dense structures, while the smoother background comes from lower density matters probably ionised.

Striking spatial variations of the infrared colour $(5-8.5 \mu \mathrm{m} / 12-18 \mu \mathrm{m})$ are also detected. Spectroscopic observations show that they are due to variations of the intensity of the aromatic features (especially at $7.7 \mu \mathrm{m}$ ) relative to a continuum emission increasing in intensity towards longer wavelengths. The contribution of the continuum relative to the aromatic features appears significantly higher at the illuminated surfaces of dense structures than in lower density matter. This effect could be the signature of the evolution of the very small particles from shielded molecular material to photo-dissociated and photo-ionised matter. We also show that size segregation due to grain dynamics in uni-directional radiation fields may play a major role.
\end{abstract}

Key words. methods: data analysis - ISM: individual objects: Orion - ISM: dust, extinction - ISM: clouds ISM: structure - infrared: ISM

\section{Introduction}

Spectroscopic observations conducted with ISO (using SWS, ISOPHOT and ISOCAM) have shown that the aromatic features at $6.2,7.7,8.6,11.3$ and $12.7 \mu \mathrm{m}$ characteristic of $\mathrm{C}-\mathrm{C}$ and $\mathrm{C}-\mathrm{H}$ bonds in aromatic hydrocarbons are ubiquitous in the interstellar medium (ISM) (Boulanger et al. 1998a; Mattila et al. 1996; Moutou et al. 1998; Uchida et al. 2000; Chan et al. 2001). The intensity of these features globally scales with the UV radiation field

Send offprint requests to: A. Abergel, e-mail: abergel@ias.u-psud.fr (for $G_{0}$ ranging $\sim 1$ to $\sim 10^{4}$, where $G_{0}$ is the average insterstellar radiation field, see Habing 1968), while their shape and relative amplitude appear remarkably constant (Boulanger et al. 1998a; Uchida et al. 2000). This is expected for emission from very small particles transiently heated to high temperature each time they absorb a UV photon (Léger \& Puget 1984; Sellgren et al. 1985).

Weak spectral features are also detected at 13.6 and $14.2 \mu \mathrm{m}$ (attributed to phenyl groups) and $16.4 \mu \mathrm{m}$ (Moutou et al. 1998, 2000; van Kerckhoven et al. 2000, see also the review by Tielens et al. 2000). However, the emission longward of $\sim 13 \mu \mathrm{m}$ is systematically dominated by 
a continuum-like emission. For very excited objects (typically with $G_{0}$ above $10^{4}$ ) this continuum is due to warm dust in thermal equilibrium with the radiation field. On the other hand, the ISO spectra of low excitation regions $\left(G_{0}=1-10^{3}\right)$ also present a continuum increasing towards long wavelengths which is due to transiently heated particles. This continuum could be a real continuum or the superpostion of unidentified spectral features. This last hypothesis is suggested by the SWS spectra of the two low excitation PDRs NGC 2023 and NGC 7023, which dip to zero around $19 \mu \mathrm{m}$ (Verstraete et al. 2001).

At the present time no experimental spectrum taken in the laboratory completely matches the observations. Thus the physical nature and the origin of the transiently heated particles in the ISM is still a matter of debate. The infrared bands were attributed to Polycyclic Aromatic Hydrocarbons (PAHs) (Léger \& Puget 1984; Allamandola et al. 1985) or aromatic sub-structures in hydrogenated amorphous carbons (Borghesi et al. 1987), in quenched carbonaceous composites (Sakata et al. 1984), in coal grains (Papoular et al. 1989) and more recently in nanodiamonds (Jones \& d'Hendecourt 2000). In any case, to be heated to a temperature of a few hundred $\mathrm{K}$ by absorption of a single UV photon, the particles need to have less than a few thousand atoms (Schutte et al. 1993). In low excitation objects, this requirement applies both to bands and continuum. Laboratory spectra exist on the one hand for molecules with few tens of atoms (e.g. Joblin et al. 1995) and on the other hand for solid particles (e.g. Guillois et al. 1996). In the case of molecules there is no continuum, while in the case of solid particles, the band to continuum contrast is very weak. The problem of the interpretation of interstellar spectra is that we do not know how to interpolate between these two extremes. The continuum is likely to be due to the solid nature of the emitters, and as a consequence to particles which are on average larger than the particles responsible for the aromatic emission bands. The same kind of particles, but with different sizes, could make both the aromatic emission and the continuum emission. The aromatic emission and the continuum emission could also be due to different families of particles, as suggested by Cesarsky et al. (2000) which propose that the continuum is due to extremely small non aromatic carbonaceous grains.

The origin of very small particles in the ISM is also an open question. Aromatic particles were believed to be formed in the envelopes of carbon stars. However, theoretical studies show that aromatic molecules are unlikelly to be generated in the stellar outflows of carbon stars (Cherchneff et al. 1992). They might also be formed during the planetary nebula phase, from carbonaceous particles condensed during the carbon-rich asymptotic giant branch phase, as suggested by Schnaiter et al. (1999). However no aromatic emission features are detectable in old carbonrich planetary nebulae such as the Helix (Cox et al. 1998), indicating that the aromatic particles, if they were formed, have been destroyed. Alternatively, the very small particles might be formed in the ISM. In order to explain spatial variations of the IRAS $12 \mu \mathrm{m} / 100 \mu \mathrm{m}$ ratio at the surfaces of dense clouds, Boulanger et al. (1990) and Bernard et al. (1993) have proposed that very small particles are photo-detached from the mantles of larger grains at the surfaces of dense clouds while in the shielded environment of dense clouds they coagulate onto grains. In that scenario, large variations of the abundance and of the optical properties of the very small particles are expected at the surfaces of dense clouds. However, IRAS had neither the sensitivity, nor the spatial and spectral resolutions for a detailed analysis of the surfaces of dense clouds which present very high brightness contrasts on small angular scales.

This paper presents the broad-band mapping of the southern part of Orion B conducted with ISOCAM (Cesarsky et al. 1996) in the $L W 2$ and $L W 3$ filters (5-8.5 $\mu \mathrm{m}$ and $12-18 \mu \mathrm{m}$, respectively) and with an angular resolution of $6^{\prime \prime}$. The field which is observed is centered on the reflection nebula NGC 2023 and contains most of the molecular cloud L1630, including the Horsehead nebula. We also present imaging spectroscopy $(5-17 \mu \mathrm{m})$ of the northern part of NGC 2023. For most of the field the far-UV illuminating flux $G_{0}$ is below $\sim 1000$. Therefore the infrared emission measured by ISOCAM is due to transiently heated small particles. Morever the spectral band pass of the $L W 2$ and $L W 3$ filters allows the separation of the spatial distribution of the aromatic and continuumlike emissions.

The paper is organised as follows. In Sect. 2 we describe the field selected in the southern part of Orion B for ISOCAM broad-band observations, then in Sect. 3 the observation strategy and the data reduction (details are given in Appendix A). In Sect. 4 we discuss the spatial structure and the colour of the infrared emission and in Sect. 5 the spectroscopic observations of the northern edge of NGC 2023 taken with the Circular Variable Filter (CVF) of ISOCAM. In Sect. 6 we discuss the different physical processes which can explain the variations of the emitting properties we see in our data. The main results are summarized in Sect. 7.

\section{Morphology and heating of the Orion B complex}

Orion $\mathrm{B}$ is the northern complex of the active OB star forming region in the Orion-Monocerus giant molecular cloud. It extends over $19 \mathrm{deg}^{2}$ and has a total mass of $\sim 10^{5} M_{\odot}$ (Kutner et al. 1977; Maddalena et al. 1986; Bally et al. 1991). The $35^{\prime} \times 40^{\prime}$ field observed by ISOCAM (Figs. 1 and 2) is centered on the reflection nebula NGC 2023, and is limited to the north by the southern edge of the star forming region NGC 2024. It contains the darkest regions of the molecular cloud L1630, including the Horsehead Nebula B33 and the optical bright nebula IC434 along the western edge of L1630. NGC 2023 and NGC 2024 are two of the four star-forming clusters in Orion B. Li et al. (1997) looked for recent star formation activity in the southern part of L1630. They found that 


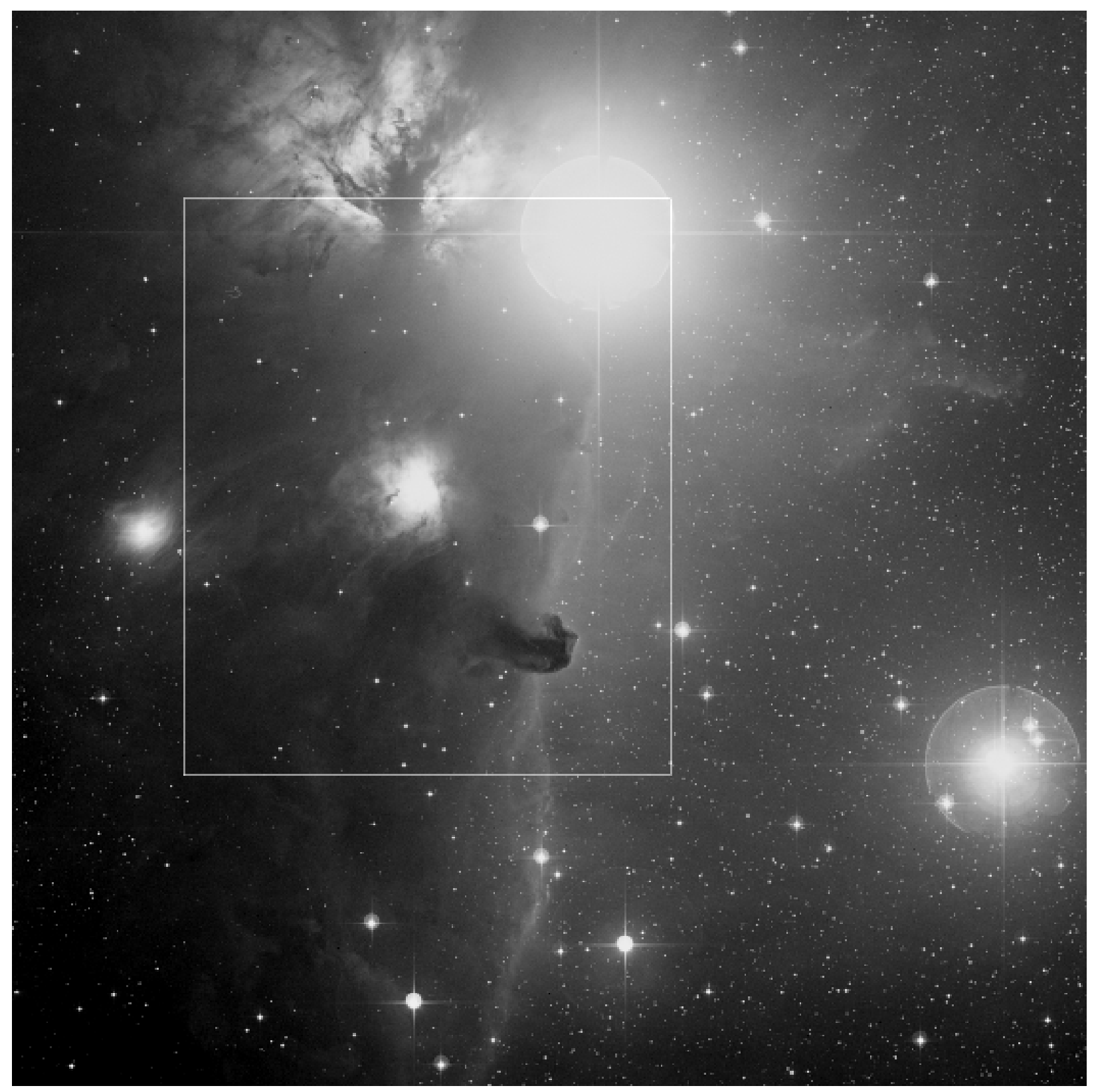

Fig. 1. The region around the molecular cloud L1630 in the visible (photographic data obtained using the UK Schmidt Telescope and extracted from the Digitized Sky Survey produced at the Space Telescope Science Institute). The white rectangle shows the $35^{\prime} \times 40^{\prime}$ field covered with ISOCAM. The large over-exposed region is centered at the OB-system $\zeta$ Ori which is located $\sim 100 \mathrm{pc}$ in front of L1630 (see the text). The edge of L1630 is essentially heated by the O9.5 V $\sigma$ Orionis system (the bright source near the western edge of the map).

outside NGC 2023, most of the infrared sources have no near infrared excess and likely correspond to field and giant stars, or stars still associated with the cloud which formed long time ago. For this paper we adopt a distance of $400 \mathrm{pc}$ to the Orion B complex which is the generally adopted distance for L1630 deduced from the study of the distances to B stars in the Orion association (390$415 \mathrm{pc}$ ) by Anthony-Twarog (1982). The illuminating star of NGC 2023 (HD 37903) is found to be at a distance of $470 \pm 290$ pc from Hipparcos data (Perryman et al. 1997). At a distance of $400 \mathrm{pc}, 1^{\prime}$ corresponds to $0.12 \mathrm{pc}$.
The two OB-systems $\zeta$ Ori to the north and $\sigma$ Ori to the west can a priori illuminate the edge of the molecular cloud from the north and the west (Reipurth \& Bouchet 1984; see Fig. 1). However Malin (1987) suggested that $\zeta$ Ori is located in the foreground and does not participate in the heating of the complex. This is confirmed by Hipparcos estimates of the distance (Perryman et al. 1997): $352 \pm 110 \mathrm{pc}$ for $\sigma$ Ori, $250 \pm 50 \mathrm{pc}$ for $\zeta$ Ori.

The western edge of L1630 presents a large scale Photo Dissociation Region (PDR) facing $\sigma$ Ori, at a projected distance of $\sim 0.5^{\circ} . \sigma$ Ori is a $09.5 \mathrm{~V}$ star (Warren \& Hesser 1977), with an effective temperature of $\sim 33000 \mathrm{~K}$ 

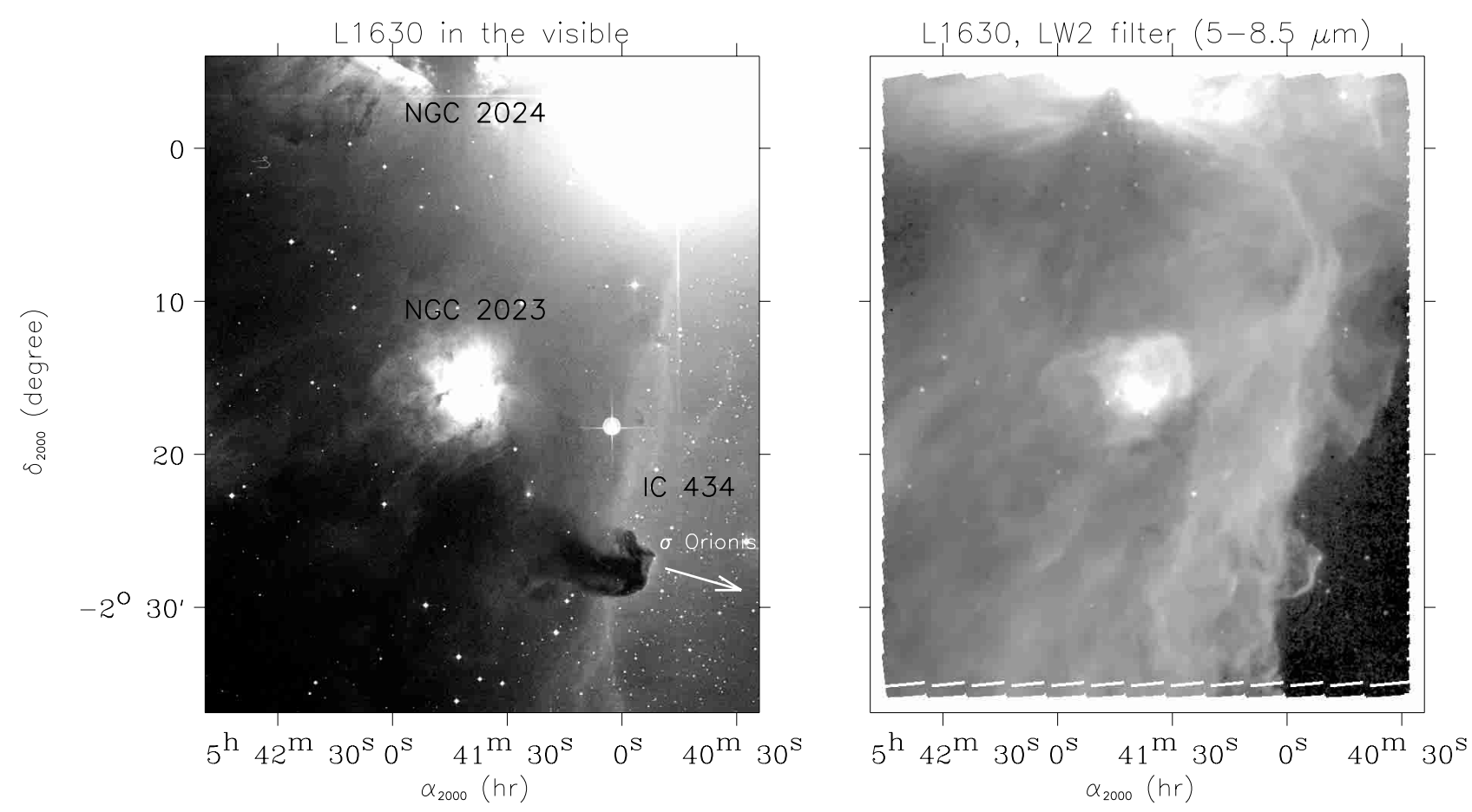

Fig. 2. Left: L1630 in the visible (extracted from Fig. 1). The white arrow gives the projected direction of the $\sigma$ Orionis system. Right: ISOCAM map within the $L W 2$ filter (in logarithmic scale).

(Panagia 1973). The incident far-UV flux $\left(G_{0}\right)$ is around 100. Due to the distance from the illuminating source, the incident flux is moderate compared to those of classical PDRs illuminated by $\mathrm{O}$ stars (generally $G_{0} \sim 10^{4}-10^{5}$ ). In front of the western illuminated edge of L1630, the true colour visible plate is dominated by red emission due to $\mathrm{H}_{\alpha}$ line emission emerging from the HII region IC 434 (e.g. Malin 1987).

The bright reflection nebulae NGC 2023 is illuminated by the embedded B $1.5 \mathrm{~V}$ star HD 37903 (Gatley et al. 1987). $G_{0}$ is estimated to be 1200 at a distance of $1^{\prime}$ (Verstraete et al. 2001) from the star. We see that for most of the field observed by ISOCAM, the radiation field is limited $\left(G_{0}<10^{4}\right)$. It has been suggested by Léger \& Puget (1984) and Sellgren et al. (1985), and confirmed recently by Boulanger et al. (1998a) from ISOCAM observations that in such environments the mid-infrared emission is due to very small particles which are transiently heated each time they absorb a UV photon. Only to the north of our field, in the region of NGC 2024, is the radiation field significantly higher $\left(G_{0} \sim 10^{4}\right.$, see Barnes et al. 1989 and Jaffe et al. 1994), so that emission due to dust at, or close to, thermal equilibrium with the radiation field should signficantly contribute to the emission measured by ISOCAM. However, in this paper we only study the emission of transiently heated particles, so we exclude the region around NGC 2024 from our analysis.

\section{Observational strategy and data reduction}

\subsection{Broad-band mapping}

The observations are part of the ISO survey of nearby star forming regions (Nordh et al. 1998). A $35^{\prime} \times 40^{\prime}$ field has been mapped using the two broad-band filters $L W 2$ and $L W 3 \quad(5-8.5$ and $12-18 \mu \mathrm{m}$, respectively). The observations have been successively conducted for the two filters. The original data, processed by ISO's automated off-line pipeline, can be imported from the ISO Data Archive (URL: http://www. iso.vilspa.esa.es/ida/index.html), catalogued under the identification TDT: 69102355. The total observing time was $\sim 8000 \mathrm{~s}$. The observational strategy and the data reduction are described in Appendix A. The maps we have computed are the best ones which can be obtained at the present time (see Fig. 2), since all the instrumental defects that we understand have been corrected or flagged (using the new processing techniques developped for ISOCAM data by Miville-Deschênes et al. 2000). These maps can be obtained from the active ISO archive. For the two filters, the photometric accuracy for the extended emission is $\sim 10 \%$, while the high frequency noise per pixel is $\sim 0.15 \mathrm{MJy} \mathrm{sr}^{-1}$. The two maps still contain defects due to bright small scale structures (especially strong point sources and filaments in NGC 2023 and NGC 2024) which produce memory effects still not properly corrected. Morever, for the sky position containing the brightest filament of NGC 2024 (also the brightest point of the covered field), some stray light is visible at a typical level of $0.1 \%$ of the bright filament.

The zodiacal emission at the time of the observations is estimated using the model of Kelsall et al. (1998): 3.8 and $25.9 \mathrm{MJy} \mathrm{sr}^{-1}$ for the $L W 2$ and $L W 3$ filters, respectively, with a spatial variation on our field of $\sim 1 \%$. The minimal emission of the ISOCAM field is measured in front 
of the Horsehead nebula, around the position $\Delta \alpha=-15^{\prime}$, $\Delta \delta=-15^{\prime}$ of Fig. 3. In this region, no significant structure emerging from the noise can be detected. Within the $L W 2$ filter, the value of the minimal emission, $5 \pm 0.5 \mathrm{MJy} \mathrm{sr}^{-1}$, is significantly above the zodiacal emission $\left(3.8 \mathrm{MJy} \mathrm{sr}^{-1}\right)$. We conclude that a uniform emission of $1.2 \pm 0.5 \mathrm{MJy} \mathrm{sr}^{-1}$ is detected within the $L W 2$ filter in front of the illuminated edge of L1630. For the $L W 3$ filter, no significant emission can be detected, since the minimal emission is $25 \pm 2.5 \mathrm{MJy} \mathrm{sr}^{-1}$, giving an emission coming from the front side of the Horsehead nebula of $-0.9 \pm 2.5 \mathrm{MJy} \mathrm{sr}^{-1}$. We see that, in the $L W 3$ filter, the uncertainty on the photometric accuracy (due to the uncertainty on the zodiacal emission model and the uncertainty on the calibration measurements, see annexe A) prevents the detection of weak extended emission which may come from the external parts of the complex. Therefore it will not be possible to discuss the properties of the emission in the faintest regions of the field. For the quantitative analysis of the emission of the bright structures, we subtract from the whole maps the minimal emissions ( 5 and $25 \mathrm{MJy} \mathrm{sr}^{-1}$ for the $L W 2$ and $L W 3$ filters, respectively). The image obtained for the $L W 2$ filter is presented in Fig. 2. A composite image is shown in Fig. 3, where the $L W 2$ and $L W 3$ emissions are codded in blue and red, respectively.

\subsection{Imaging spectroscopy}

A $3^{\prime} \times 3^{\prime}$ field north of NGC 2023 has also been observed using the Circular Variable Filters (CVFs) of ISOCAM (see Fig. 4 for the foot print of the field on the larger raster map area). The original data are catalogued under the identification TDT: 68701905 in the ISO Data Archive. The full wavelength range of the CVFs was used: the filter wheel was stepped starting at the shortest wavelength, $5.02 \mu \mathrm{m}$, all the way up to $16.98 \mu \mathrm{m}$. The number of wavelengths observed is 161 , with a resolution of approximately 40. The elementary integration time per detector readout was $2.1 \mathrm{~s}$ and 10 readouts were obtained per CVF step, the total observing time was of the order of $3720 \mathrm{~s}$. The data was "deglitched", dark corrected and transient corrected as explained in Appendix A. In NGC 2023 the emission is extended. In that case, some straylight is known to degrade the images and bias the photometry of CVF observations by factors up to $\sim 30 \%$ depending on the wavelengths (Blommaert et al. 2001a) and the spatial structure of the emission. Unfortunately, there is no meaningful straylight correction for regions containing extended emission with contrasted structures (Blommaert et al. 2001b), which is the case in our field. Therefore we have used the standard spectral response function measured during the mission using photometric stars (Blommaert et al. 2001a), without any correction of the degradations which may be due to straylight.

\section{ISOCAM images of L1630}

On large angular scales the infrared emission appears, to first order, to be anti-correlated with the visible emission (Fig. 2). In the visible, several structures are seen in extinction at the illuminated edge of L1630, in front of the extended emission of the HII region IC 434 . The best example is obviously the Horsehead nebula which emerges from its parental molecular cloud due to the eroding UV radiation emitted by $\sigma$ Ori. The bright region at the center of L1630 corresponds to the reflection nebula NGC 2023. The northern limit of the ISOCAM image coincides with the very bright ionisation front at the southern part of NGC 2024 (e.g. Barnes et al. 1989).

We have shown in Abergel et al. 2000b (see also Abergel et al. 2002b) that the edge of the Horsehead nebula presents the sharpest $\left(\sim 10^{\prime \prime}\right.$, or $\left.\sim 0.01 \mathrm{pc}\right)$ filament detected by ISOCAM in the complex. This filament is due to the combined effect of steep density gradients and extinction: the increase of emission on the illuminated side is due to a steep increase of column density, while on the other side the decrease of the emission is due to the extinction of the incident radiation by dense material. Thus, the infrared filament corresponds to the illuminated surface of the dark cloud observed edge-on. The ISOCAM images reveal sharp structures down to the angular resolution $\left(6^{\prime \prime}\right)$ with an amplitude significantly higher than the noise per pixel $\left(\sim 0.15 \mathrm{MJy} \mathrm{sr}^{-1}\right)$. We suggest that, as the filament at the edge of the Horsehead nebula, most of the sharp structures correspond to illuminated surfaces of dense structures. This scenario could explain simply the fact that many bright filaments appear elongated perpendicular to the illuminating direction (e.g., compare in Fig. 2 the projected direction of the $\sigma$ Ori star with the orientation of the filament-like structures inside L1630). This is an hypothesis which still has to be confirmed using molecular observations to trace the spatial variations of the local density at small angular scales.

Strong spatial variations of the infrared colour are visible in the composite image (Fig. 3). The value of the $I_{\nu}(L W 2) / I_{\nu}(L W 3)$ ratio for the sharp brightness fluctuations (see the brightness profiles shown in Fig. 3 ) is $\sim 0.8$ (with a relative uncertainty due to the noise better than $10 \%$ ), which corresponds to red emission on the composite image of Fig. 3. We suggested above that most of the sharp structures inside the complex are due to the illuminated surfaces of dense clouds. In this scenario, illuminated surfaces appear to have a value of the $I_{\nu}(L W 2) / I_{\nu}(L W 3)$ ratio of $\sim 0.8$. The best example is obviously the bright filament at the edge of the Horsehead nebula (see in the middle panel of Fig. 3 the brightness profiles at $\Delta \delta=$ $\left.-12.5^{\prime}\right)$.

The small scale brightness fluctuations across L1630 appear on top of a large scale extended emission with a $I_{\nu}(L W 2) / I_{\nu}(L W 3)$ ratio of $\sim 2.5$ (with a relative uncertainty better than $\sim 10 \%$ ), corresponding to blue emission in the composite image (Fig. 3). As this blue emission contains little small scale fluctuations, we suggest that it is essentially emitted by particles located in low density regions surrounding the dense parts of the complex (which present steep density gradients). Strong variations of the infrared colour are also found in NGC 2023 (Fig. 4). They 

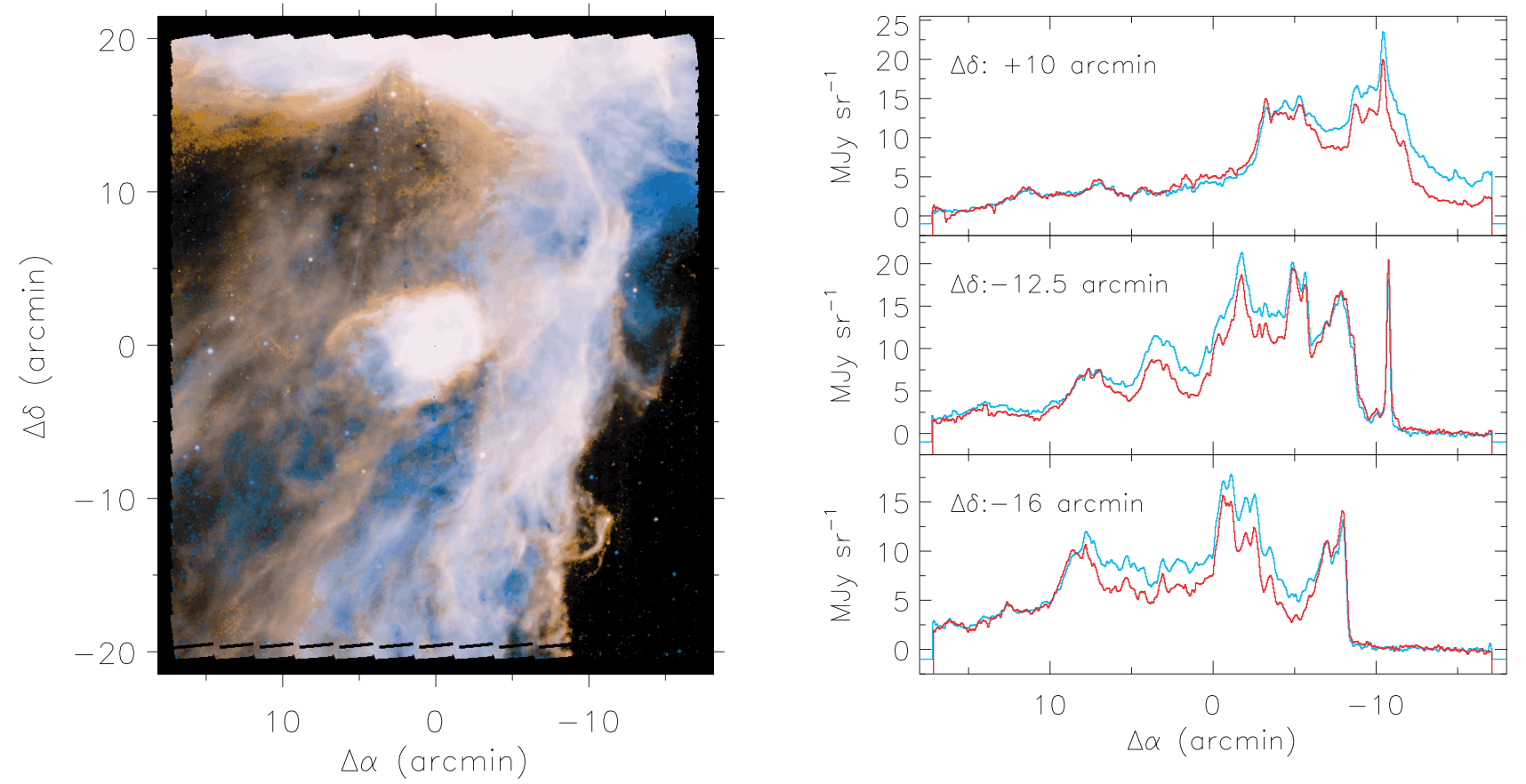

Fig. 3. Left: composite image of the ISOCAM emission. The $L W 2$ and $L W 3$ emissions are codded in blue and red, respectively. The reference position $\left(\Delta \alpha=\Delta \delta=0^{\prime}\right)$ corresponds to $\alpha=5^{\mathrm{h}} 41^{\mathrm{m}} 37^{\mathrm{s}}, \delta=-2^{\circ} 15^{\prime} 26^{\prime \prime}$. The central region (NGC 2023) is saturated because of the dynamic range selected for this figure (to show the colour variations in this region, see Fig. 4 which presents the ratio of the $L W 2$ and $L W 3$ emissions). The position of the main illuminating source of L1630 (the O $9.5 \mathrm{~V} \sigma$ Ori system) is at the position $\Delta \alpha=-43.3^{\prime}, \Delta \delta=-20.6^{\prime}$. Right: brightness profiles at 3 constant declinations ( $L W 2$ : Blue, $L W 3$ : Red). The profile at $\Delta \delta=-12.5^{\prime}$ crosses the bright filament at the edge of the Horsehead nebula (see the text in Sect. 4).

are discussed in the next section, together with spectroscopic observations.

\section{Spectroscopic observations at the northern edge of NGC 2023}

The CVF observations of the field north of NGC 2023 (Fig. 4) were performed with the same angular resolution $\left(6^{\prime \prime}\right)$ as that used for the $L W 2$ and $L W 3$ observations. Spectral line features and continuum emission are detected on each of the $32 \times 32$ spectra. We have decomposed each spectrum using Lorentzian profiles for the aromatic features, following the method described in Boulanger et al. (1998b). The $S(1), S(2), S(3)$ and $S(5)$ pure rotationnal lines of molecular hydrogen are also detected and fitted using Gaussian profiles. The continuum is fitted with a second order polynomial. We have to keep in mind that this decomposition is not unique, especially for the continuum. In particular, we have checked that comparable agreements are found with broad lines centered at $\sim 10 \mu \mathrm{m}$ and $\sim 15 \mu \mathrm{m}$ and a negligible continuum.

Figure 5 presents examples of the fitted spectra for two selected pixels with low and high values of the $I_{\nu}(L W 2) / I_{\nu}(L W 3)$ ratio $(0.6$ and 1.7 , respectively, with a relative uncertainty due to the noise of $1 \%)$. Strong variations of the band to continuum constrats are visible, with an amplitude $(\sim 2)$ larger than what could be expected from straylight effects (see Sect. 3.2). The SWS spectrum obtained in NGC 2023 by Verstraete et al. (2001), at the position shown in Fig. 4, is also shown for comparison. The photometric accuracy of this spectrum is $\sim 15-25 \%$ (Leech et al. 2001). Within the SWS slit we have $I_{\nu}(L W 2) / I_{\nu}(L W 3) \sim 1.5$. The shape of the CVF spectrum for the pixel with $I_{\nu}(L W 2) / I_{\nu}(L W 3)=1.7$ (middle panel of Fig. 5) and of the SWS spectrum appears comparable, which shows that straylight effects do not strongly affect the general shape of the CVF spectra.

For each pixels, the intensity of the $7.7 \mu \mathrm{m}$ feature is computed from the Lorentzian fitting of the line. Images of the $7.7 \mu \mathrm{m}$ feature intensity and the $15 \mu \mathrm{m}$ continuum are compared to broad band images in Fig. 6. We see that the emission within the $L W 2$ filter is spatially correlated with the $7.7 \mu \mathrm{m}$ feature while the emission within the $L W 3$ filter is correlated with the $15 \mu \mathrm{m}$ continuum. This result confirms that the emission within the $L W 2$ filter measures the intensity of the aromatic bands while the emission within the $L W 3$ filter is that of the continuum. As a consequence, the $L W 2 / L W 3$ map are correlated with the intensity ratio of the $7.7 \mu \mathrm{m}$ feature $/ 15 \mu \mathrm{m}$ continuum (two lower panels of Fig. 6).

The decrease of $I_{\nu}(L W 2) / I_{\nu}(L W 3)$ from $\sim 1.7$ to $\sim 0.8$, for increasing distance from the illuminating star, is due to the increasing contribution of the continuum relative to the aromatic bands. The illuminating star HD 37903 is located in a low density cavity of photodissociated matter. The CVF observations of NGC 2023 suggest that the emission with $I_{\nu}(L W 2) / I_{\nu}(L W 3) \sim$ 0.8 is characteristic of illuminated surfaces of dense structures, while $I_{\nu}(L W 2) / I_{\nu}(L W 3) \sim 1.7$ is obtained for lines of sight in the central parts of NGC 2023 crossing the low density cavity around the illuminating 

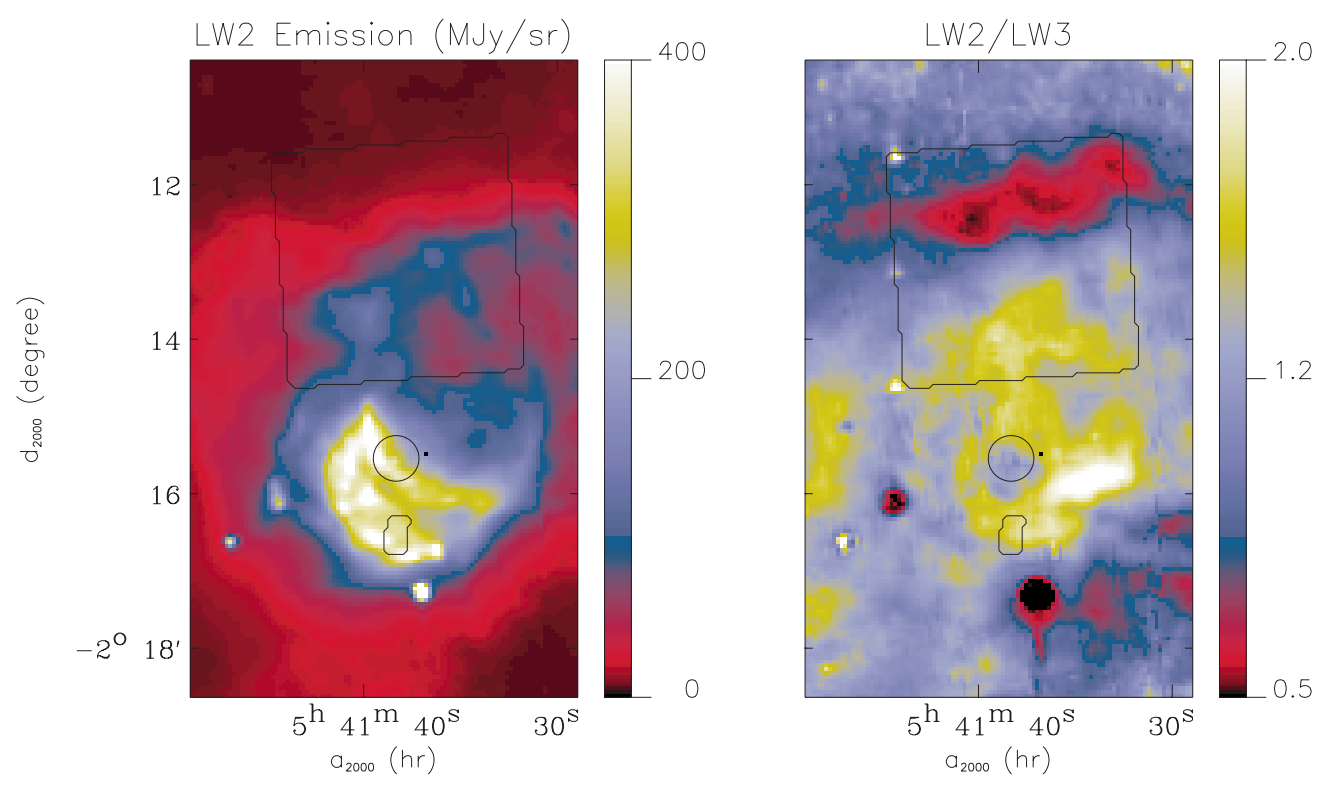

Fig. 4. ISOCAM emission measured around NGC 2023. The circle is centered at the position of the B $1.5 \mathrm{~V}$ illuminating star HD 37903. The square and the rectangle show the field covered by the CVF observations and the slit of the SWS (Verstraete et al. 2001), respectively.

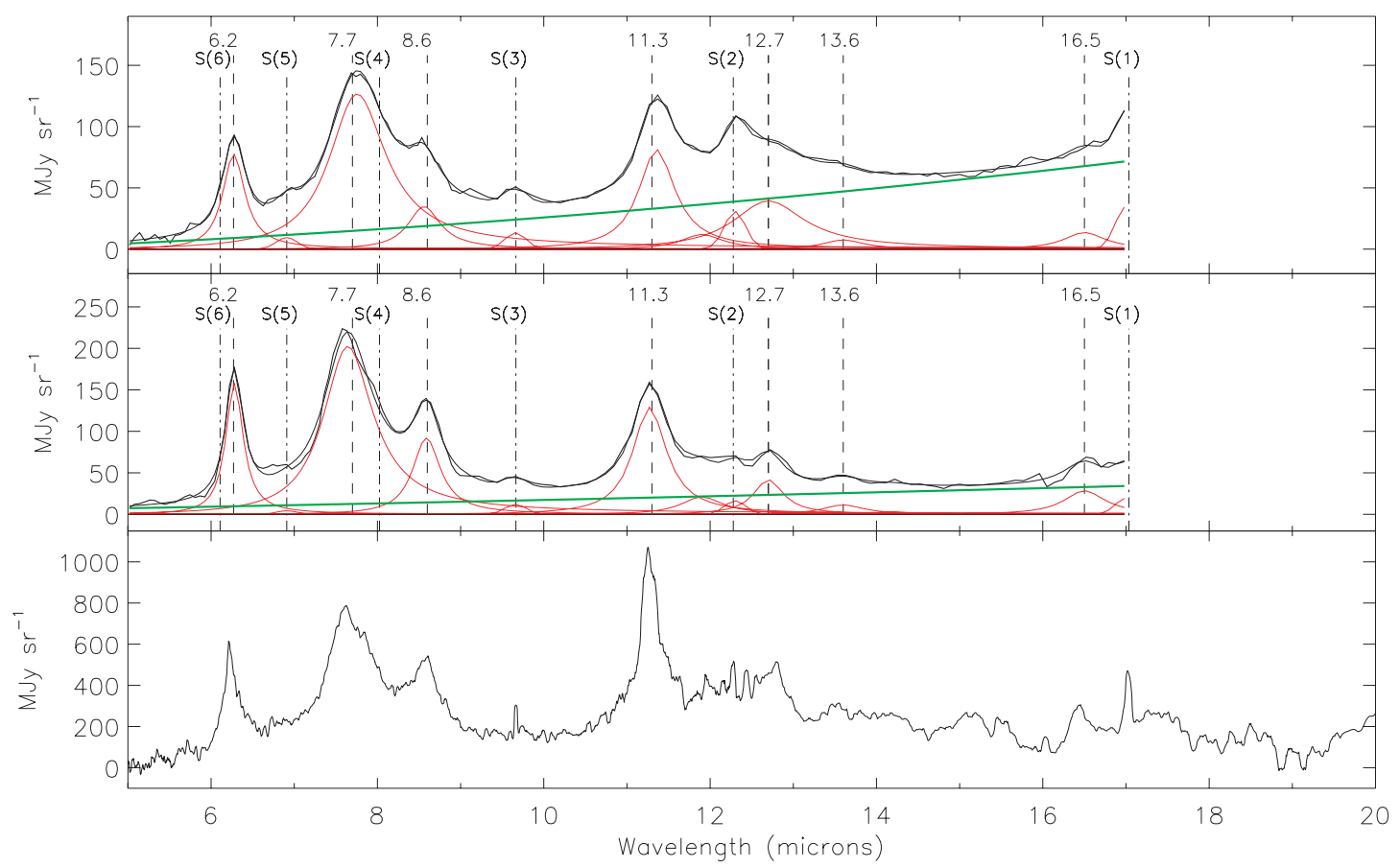

Fig. 5. Some illustrative spectra in NGC 2023: CVF spectra for a pixel with $I_{\nu}(L W 2) / I_{\nu}(L W 3)=0.6$ and $I_{\nu}(L W 2) / I_{\nu}(L W 3)=$ 1.7 (top and middle panels, respectively), and SWS spectrum (bottom panel, from Verstraete et al. 2001). For the CVF spectra, the aromatic lines are fitted with Lorentzian profiles, following Boulanger et al. (1998b). The $S(1), S(2)$ and $S(3)$ pure rotationnal lines of molecular hydrogen are adjusted with Gaussian profiles. The other rotationnal lines are marginally detected for some pixels.

star. The geometry of NGC 2023 is such that this last value is an "average" between the illuminated surface value $\left(I_{\nu}(L W 2) / I_{\nu}(L W 3) \sim 0.8\right)$ and that of matter inside the low-density cavity $\left(I_{\nu}(L W 2) / I_{\nu}(L W 3) \sim 2.5\right)$.

\section{Interpretation of the colour variations}

We conclude from the previous two sections that the colour variations found both across L1630 and in
NGC 2023 are comparable. At the illuminated surfaces of dense structures, low values of $I_{\nu}(L W 2) / I_{\nu}(L W 3)$ are found $(\sim 0.8)$, while high values $(\sim 2.5)$ are detected in lower density photo-dissociated matter. Low values of $I_{\nu}(L W 2) / I_{\nu}(L W 3)$ are due to a relatively strong continuum emision around $15 \mu \mathrm{m}$ compared to the intensity of the aromatic features, and high values to a relatively small 

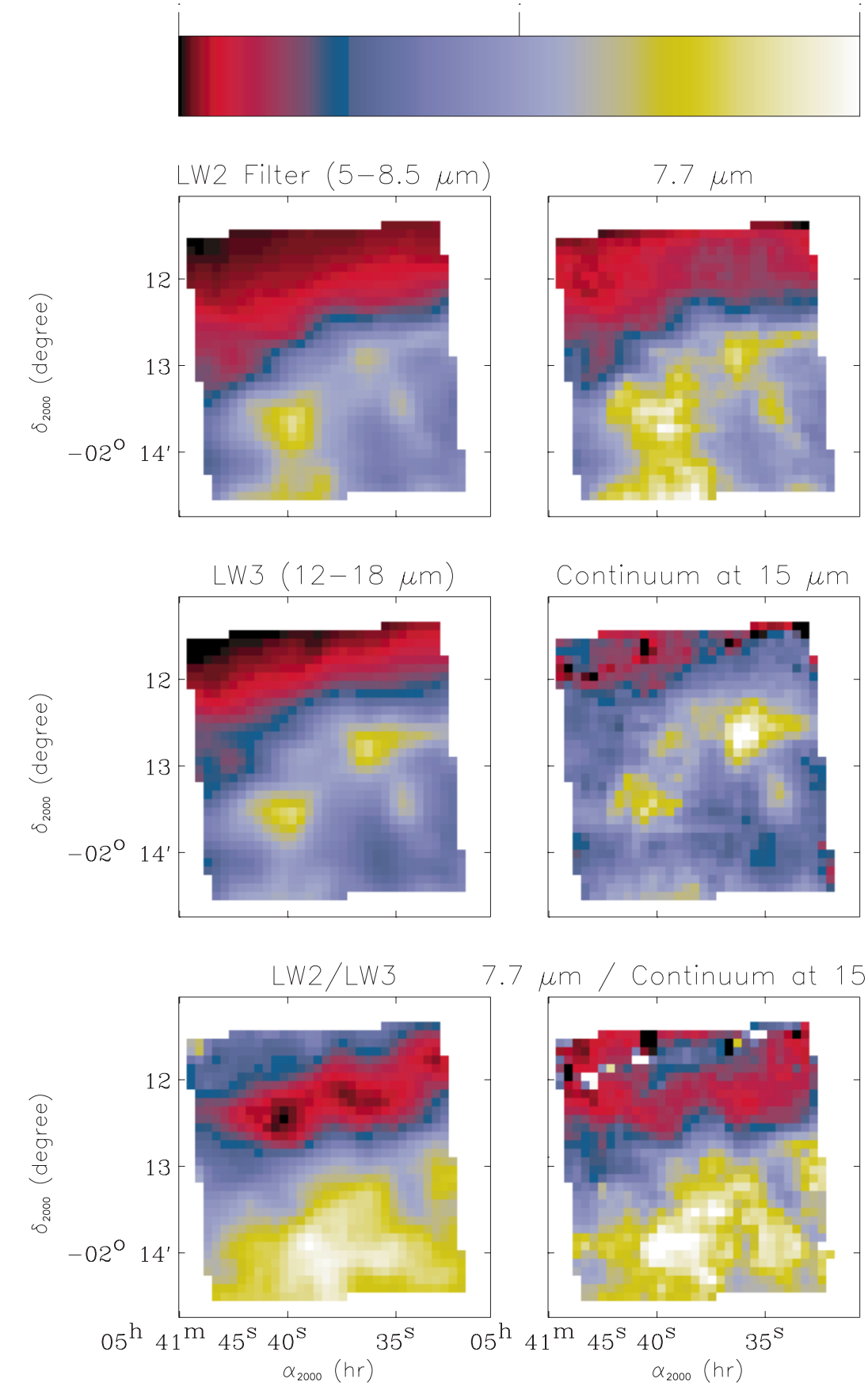

Fig. 6. Comparison of the spatial distribution of $L W 2$ and $L W 3$ emissions of the northern edge of NGC 2023 with the intensity of the $7.7 \mu \mathrm{m}$ feature and of the continuum at $15 \mu \mathrm{m}$. The right panels present ISOCAM-CVF observations, the left panels broad-band ISOCAM observations within the $L W 2$ and $L W 3$ filters. The $L W 2$ emission appears perfectly correlated with the $7.7 \mu \mathrm{m}$ feature, while the $L W 3$ emission is correlated to the continuum at $15 \mu \mathrm{m}$.

continuum emision around $15 \mu \mathrm{m}$ compared to the intensity of the aromatic features.

Comparable results are found by Cesarsky et al. (2000) in the reflection nebula Ced 201, located at the edge of a molecular cloud and illuminated by a cold star (B9.5V). In a transparent cloud $\left(A_{V}<0.5\right)$, Miville-Deschênes et al. (2002) have observed the same tendancy for the variations of the ratio $I_{\nu}(L W 2) / I_{\nu}(L W 3)$ with the local density (from low density atomic regions to molecular gas). They propose that these variations are due to abundance variations which are related to the production of very small particles by grain shattering in grain-grain collisions generated by turbulent motions. The physical conditions of their cloud are different from the physical conditions in L1630 and NGC 2023, so the interpretation of the colour variations is not necessarily the same.

Using a typical infrared spectrum of this region (the SWS spectrum of NGC 2023 shown in the lower panel of Fig. 5, from Verstraete et al. 2001) and the Draine \& Lee (1984) extinction law, we have checked that the 
variations of the ratio $I_{\nu}(L W 2) / I_{\nu}(L W 3)$ we observe cannot be due to infrared extinction (typical values of $A_{V}$ across L1630 are in the range $1-5$ ). They reveal intrinsic variations of the spectrum emitted by the very small particles, without any radiative transfer effects in the infrared. Unfortunately, we cannot connect these colour variations to variations of the absolute emissivity of the particles, because we have no observation of an independant tracer of the total column density (e.g. the $100 \mu \mathrm{m}$ emission or $A_{V}$ ) taken at a sufficient angular resolution. The illuminated edges of L1630 and NGC 2023 are strongly illuminated by $\mathrm{O}$ and B stars with $G_{0} \sim 100$ and $G_{0} \sim 1000$, respectively. Therefore the colour variations we detect could be due to photo-processing effects of the emitting particles.

\subsection{Change in emission properties and/or particles nature}

The colour variations could trace changes of the emission properties of small dust particles. An example of this is the effect of ionisation of PAH molecules on their midinfrared emission. In the case of NGC 2023, and within the framework of the free PAH model, Dartois \& d'Hendecourt (1997) have shown that the fraction of cations decreases from 1 outside the cloud to 0 inside the cloud, on a scale of typically $0.1 \mathrm{pc}$ (or $1^{\prime}$ ). Laboratory data and quantum calculation show that the ionisation of free PAHs in cations strongly enhance the emissivity of the C-C stretching $(6.2$ and $7.7 \mu \mathrm{m})$ and the $\mathrm{C}-\mathrm{H}$ in plane bending $(8.6 \mu \mathrm{m})$ features, with respect to the intensity of the C-H out-of-plane bending features at 11.3 and $12.7 \mu \mathrm{m}$ (Hudgins \& Allamandola 1995; Szczepanski \& Vala 1993; Langhoff 1996; Hudgins \& Sandford 1998). However, the spectra we have toward NGC 2023 indicate that the ratio $I(7.7) / I(11.3)$ does not significantly vary at the illuminated edge (compare the two CVF spectra presented in Fig. 5). More generally, the strong effects of ionisation on the relative intensity of the aromatic features predicted by the free PAH model are not detected in the infrared spectra of PDRs (e.g. Uchida et al. 2000). Therefore there is no indication that the spatial variations of the ratio $I_{\nu}(L W 2) / I_{\nu}(L W 3)$ that we see in our field could be due to variations of the emitting properties arising from the ionisation state. Note that the $I_{\nu}(L W 2) / I_{\nu}(L W 3)$ colour is high for the large scale emission around L1630 which we believe is associated with ionised gas where PAHs due to the high electron densities are expected to be neutral. But UV radiation can have more complex impact on matter.

At the illuminated surfaces of dense structures, the UV radiation could transform the particles making the continuum into aromatic particles which may survive (Allain et al. 1996 have shown that PAHs with typically more than $\sim 50 \mathrm{C}$ survive exposure to strong UV radiation). It has been suggested by Cesarsky et al. (2000) to explain the colour variations in the reflection nebula Ced 201. The ISOCAM observations could therefore trace the photo-processing of organic material into small refractory aromatic particles at the edges of molecular clouds.
This could result of the shattering of the photo-processed ice mantles, as suggested by Greenberg et al. (2000).

\subsection{Size segregation due to grain dynamics}

We mentionned in the introduction that the continuum emission could be due to particles which are on average larger than the particles producing the aromatic emission bands. Therefore an other explanation of the colour variations could be size segregation due to grain dynamics. At the edge of L1630 and NGC 2023 the radiation is dominated by $\sigma$ Orionis and HD 37903, respectively, and is therefore almost uni-directional. As in all anisotropic radiation fields the dust is subject to a resultant radiation pressure force, and also to recoil forces due to the photoejection of electrons, the photodesorption of weakly-bound surface species and the surface formation and ejection of molecular hydrogen. Weingartner \& Draine (2000, 2001) showed that these forces may strongly affect the grain dynamics in a given environment.

In order to understand the details and the importance of the various grain dynamical processes in anisotropically irradiated regions we have begun a detailed study of these processes. In the preliminary study presented in Appendix B, we ignored the effects of stellar winds, the coupling between the charged grains and the magnetic field, and the effects of desorption processes. For typical interstellar particles with sizes ranging from $1 \mathrm{~nm}$ to $100 \mathrm{~nm}$ in the region around NGC 2023, we find that the larger grains couple more efficiently to the stellar radiation field than the smaller grains through the effects of radiation pressure. This effect is due to the fact that at ultraviolet and optical wavelengths larger grains have larger values of the product of the grain geometrical cross-section and the absorption efficiency factor (e.g., Weingartner \& Draine 2001). For a given environment and radiation field it is this product that determines the efficiency of the coupling between a given grain and the radiation field. The more efficient coupling of the larger grains may produce an underabundance of large grains relative to small grains in the low density regions in NGC 2023 and the edge of L1630. In the scenario that the $L W 2$ emission is due to particles smaller than the particles making the $L W 3$ emission, this process may explain why we observe a $I_{\nu}(L W 2) / I_{\nu}(L W 3)$ ratio that is systematically lower at the illuminated surfaces of dense structures than in the low density regions surrounding the sources of illumination.

\section{Conclusions}

The ISOCAM images of the southern part of Orion B includes the reflection nebula NGC 2023 and the Horsehead nebula illuminated by the B star HD 37903 and the O star $\sigma$ Orionis, respectively. Within the two broad band 5-8.5 $\mu \mathrm{m}$ and $12-18 \mu \mathrm{m}$ filters ( $L W 2$ and $L W 3$, respectively), they reveal the spatial distribution of the emission of very small particles (with less than a few thousands atoms). Due to the limited radiation field $\left(G_{0} \leq 1000\right)$, 
these particles are transiently heated each time they absorb a UV photon. Spectroscopic observations conducted with ISO show that the emission within the $L W 2$ filter is due to aromatic features (dominated by the $7.7 \mu \mathrm{m}$ feature), while the emission within the $L W 3$ filter is due to an apparent continuum increasing towards long wavelengths.

A filamentary structure is detected at small angular scales (down to the angular resolution of $6^{\prime \prime}$ ) on top of a smoother background. A spectacular filament outlines the heated edge of the Horsehead nebula facing $\sigma$ Orioni. This particular case suggests that the filaments in general result from the illuminated surfaces of dense structures, while the smoother background comes from lower density matters probably ionised. The increase of emission on the illuminated side is due to a steep increase of column density, while on the other side the decrease of the emission is due to the extinction of the incident radiation by dense material. In the absence of an additional tracor of the column density at comparable angular resolution, one cannot exclude that part of the brightness contrast is due to changes in the abundance of small grains. Such abundance variations have been infered in cirrus clouds from the comparison of ISOCAM maps with gas observations (Miville-Deschênes et al. 2002).

Striking variations in the infrared colour $(L W 2 / L W 3)$ are detected between the filaments and the background. The contribution of the continuum relative to the intensity of the aromatic features appears significantly higher at the illuminated surfaces of dense structures than in lower density matter. This is a systematic effect found for all filaments of our map and in other fields (e.g. Abergel et al. 2002a). We propose two interpretations of the colour variations:

1. They may trace the evolution of the very small particles from shielded molecular material to photodissociated and photo-ionised matter. This evolution could affect the emission properties and the nature and abundance of the particles. It cannot be simply the expected effect of the ionisation state on the emission from PAHs. The ISOCAM observations could trace the photo-processing of organic material into small refractory aromatic particles at the edges of molecular clouds exposed to UV photons.

2. We also show that size segregation due to grain dynamics may play a major role. At the illuminated surfaces of dense clouds the radiation is almost uni-directional, and one expects the larger grains to couple more efficiently to the stellar radiation field than the smaller grains. Within the hypothesis that the particles making the continuum emission should, on average, be larger than the aromatic emitters, this could explain the observed colour variations.

Comparable colour variations are also seen within cirrus clouds where the physical conditions (overall density and radiation field) are quite different. For these clouds, Miville-Deschênes et al. (2002) proposed that the colour variations result from the impact of turbulence on the size distribution of grains. It is not clear to us if turbulence could also affect the small particle size distribution in illuminated surfaces of molecular clouds.

In any case the main difficulty in reaching any definitive conclusion is that the exact nature of the very small particles, transiently heated by UV photons, is still unknown. New laboratory data on particles comparable in size to very small intestellar grains are urgently needed.

\section{Appendix A: Observational strategy and data reduction of the broad-band observations}

To cover a $35^{\prime} \times 40^{\prime}$ field with the two broad-band filters $L W 2$ and $L W 3$ (5-8.5 and $12-18 \mu \mathrm{m}$, respectively), two identical rasters with $26 \times 13$ pointed observations on the sky were repeated. The scanning directions are parallel to the equatorial declination and right ascension directions, with $\Delta \delta$ and $\Delta \alpha$ equal to 0.46 and 0.8 times the frame size respectively. The $6^{\prime \prime}$ pixel-field-of-view lens was used, so the point spread function is under-sampled by a factor $\sim 2$. At each raster position, 60 frames were taken with the minimal integration time $(0.28 \mathrm{~s})$. For such short intergration times, frames were accumulated on board 4 by 4 . The slew time between 2 successive positions is $\sim 6.5 \mathrm{~s}$, corresponding to $\sim 23$ frames. For each filter, the whole field has been covered using 338 sky positions, the total number of transmitted frames was $\sim 28000$, and the total observing time was $\sim 8000 \mathrm{~s}$.

For each readout, a dark image is computed using the polynomial time function of Biviano et al. (1998). In the general case, the response of pixels affected by cosmic ray particles instantaneously increases ("glitch"), then recovers after one or two readouts. These events are discarded on a pixel by pixel basis at the beginning of the processing. We apply a temporal median filtering which detects all readouts deviating from the running median value by more than a threshold value (three times the current rms noise). Typically $1.4 \%$ of the data are affected. The LW array presents a time lagged response after a flux step depending on the flux history, the pixel number and the spatial structure of the illumination (Abergel et al. 2000a). We use the new model developped by Coulais \& Abergel (2000), optimised for low contrasted illumination. This model generally fails for strong point sources, but low contrasted regions are corrected with an accuracy of $\sim 1 \%$. Moreover, impacts due to heavy ions produce glitches which can affect the response of individual pixels during periods of several seconds to hours. Practically these two effects produce a relative response of the pixels of the camera ("flat-field") which fluctuates with time. We use the techniques developped by Miville-Deschênes et al. (2000) to correct our data. First we assume a constant flat-field for the whole observation extracted from each filter by computing the mean value of the lowest percentile $(10 \%)$ seen by each pixel. Then we use this flat-field to compute a first estimate of the sky map, $I_{\text {sky }, 0}(\alpha, \delta)$. We spatially smooth $I_{\mathrm{sky}, 0}(\alpha, \delta)$ on an angular size equal to the detector size, and use this smoothed sky image to derive, for 
each group of 70 successive sky positions, an optimised flat field (see details in Miville-Deschênes et al. 2000). The sky map we derive $\left(I_{\text {sky }, 1}(\alpha, \delta)\right)$ is still affected by low amplitude glitches not filtered with our deglitching method at the beginning of the processing. Therefore, we go back to the raw data cube and, using the spatial redundancy of the observations, flag the "bad pixels". We again compute the optimised time dependent flat-field variable with time, and the final sky map $I_{\text {sky }}(\alpha, \delta)$.

We use the standard absolute calibration of ISOCAM based on the observation of calibration stars (Blommaert et al. 2001a). The absolute flux calibration for point sources in the $L W 2$ and $L W 3$ filters is $\sim 5 \%$, due to photometric uncertainties on the calibration stars $(\sim 3 \%)$ and stabilization effects. In this paper we are essentially interested in extended emission. By combining observations of the zodiacal emission with the zodiacal emission model of Kelsall et al. (1998) calibrated from the DIRBE data, it is found that the extended emission calibration agrees within $\sim 10 \%$ with that of the point sources (Blommaert et al. 2001b), the discrepancy being due to the uncertainty on the zodiacal model and to the photometric accuracy of ISOCAM. Thus, the photometric accuracy in our maps for the extended emission is $\sim 10 \%$. The noise per pixel is estimated by computing the standard deviation within an empty part of the field, taken in front of the Horsehead nebula. We have obtained for the two filters a value of $0.15 \mathrm{MJy} \mathrm{sr}^{-1}$.

\section{Appendix B: Radiation pressure effects in NGC 2023}

To illustrate the effect of radiation pressure we consider the region around NGC 2023 which is dominated by the radiation field of a star $\mathrm{B} 1.5 \mathrm{~V}\left(T_{\text {eff }}=23700 \mathrm{~K}, R_{\star}=\right.$ $\left.6 R_{\odot}\right)$. The ISOCAM $L W 2$ and $L W 3$ data show that there is decreased emission from "large" dust grains ( $L W 3$ emitters) in the immediate surroundings of the star, i.e., some process or combination of processes has created a cavity in the dust distribution with the preferential removal of the large dust grains from the central cavity. Can radiation pressure effects explain this observed effect?

Using a simple one-dimensional model we can estimate the effects of radiation pressure on the dust in this region. Making the simplifying assumption that the star at the centre of the region instantly illuminates a cloud of homogeneously distributed dust, we can then make order of magnitude estimates of the likely radiation pressure effects. We assume a canonical gas density of $n_{\mathrm{H}}=100 \mathrm{~cm}^{-3}$ and isotropic dust scattering at all wavelengths (i.e., radiation pressure efficiency factor is $\left.Q_{\mathrm{pr}}=Q_{\mathrm{abs}}+Q_{\mathrm{sca}}=Q_{\mathrm{ext}}\right)$.

If $F_{\mathrm{pr}}$ is the radiation pressure force on a grain in the radiation field of a star and $F_{\text {drag }}$ is the opposing force due to the drag caused by collisions with gas in the medium surrounding the star, then the equation of motion of the grain is

$\left(F_{\mathrm{pr}}-F_{\mathrm{drag}}\right)=m_{\mathrm{g}} \frac{\mathrm{d} v_{\mathrm{g}}}{\mathrm{d} t}$

and the corresponding force terms are

$F_{\mathrm{pr}}=\pi a^{2} Q_{\mathrm{pr}}\left(\frac{\pi R_{\star}^{2}}{D^{2}} \times \frac{\lambda B_{\lambda}\left(T_{\mathrm{eff}}\right)}{c}\right)$

$F_{\text {drag }}=1.4 m_{\mathrm{H}} 1.1 n_{\mathrm{H}} \pi a^{2} v_{\mathrm{g}}^{2}$.

In Eqs. (B.1)-(B.3) $m_{\mathrm{g}}$ is the grain mass, $a$ is its radius and $v_{\mathrm{g}}$ its velocity, $D$ is the distance of the grain from the star and all other symbols have their usual meaning. In Eq. (B.3) $1.4 m_{\mathrm{H}}$ and $1.1 n_{\mathrm{H}}$ are the mean gas phase particle mass and number density.

The grain asymptotic drift velocity can then be derived by substitution of Eqs. (B.2) and (B.3) into Eq. (B.1), where the integration of $Q_{\mathrm{pr}}$ over the stellar radiation field $B_{\lambda}\left(T_{\text {eff }}\right)$ is required, and then solving for the grain velocity. Thus, the grain asymptotic drift velocity, $v_{\text {drift }}$, is given by

$v_{\mathrm{drift}}=\left(\frac{\pi R_{\star}^{2}}{1.4 m_{\mathrm{H}} 1.1 n_{\mathrm{H}} D^{2} c} \int_{\lambda_{0}}^{\lambda_{\infty}} Q_{\mathrm{pr}} B_{\lambda}\left(T_{\mathrm{eff}}\right) \mathrm{d} \lambda\right)^{\frac{1}{2}}$

Typical asymptotic drift velocities are $\approx 100 \mathrm{~km} \mathrm{~s}^{-1}$ $\left(\approx 1 \mathrm{~km} \mathrm{~s}^{-1}\right)$ for $100 \mathrm{~nm}(1 \mathrm{~nm})$ radius carbon and silicate grains $0.01 \mathrm{pc}$ from the star. For the same grains at $0.1 \mathrm{pc}$ the velocities are an order of magnitude smaller.

The calculation of $v_{\text {drift }}$ allows us to calculate the grain ejection time-scale, i.e.,

$t_{\text {eject }}=\int_{0}^{D_{\text {shell }}}\left(\frac{1}{v_{\text {drift }}(D)}\right) \mathrm{d} D$,

where $t_{\text {eject }}$ is the time to remove a grain from a distance $D$ to the dense shell surrounding the cavity (assumed to be at $0.2 \mathrm{pc}$ ). The derived grain ejection time-scales typically range from $\approx 10^{4} \mathrm{yr}$ for $100 \mathrm{~nm}$ grains to $\approx 10^{6} \mathrm{yr}$ for $1 \mathrm{~nm}$ grains. However, in assessing this ejection time-scale it is necessary to take account of the finite grain acceleration time. Assuming that the grain is accelerated only by radiation pressure the grain acceleration time-scale is given by

$t_{\mathrm{acc}}=v_{\mathrm{drift}}(D)\left(\frac{F_{\mathrm{pr}}(D)}{m_{\mathrm{g}}}\right)^{-1}$.

We find that the typical grain acceleration time-scale ranges from a few tens of years for small grains close to the star to about a thousand years for large grains in the outer cavity regions.

Thus, based on our grain ejection time-scales we show that large grains do indeed couple more effectively to the stellar radiation field and are therefore preferentially ejected from the region before the smaller grains. This holds true for the grains within $0.1 \mathrm{pc}$ of the star. However, 
the time required to accelerate the large grains to their asymptotic drift velocities increases with distance from the star. Only near the dense shell at the edge of the cavity does the acceleration time-scale approach the grain ejection time-scale for the large grains. Thus, the very simple model presented here provides a means of explaining the overabundance of very small grains ( $L W 2$ emitters), with respect to large grains ( $L W 3$ emitters), in the cavity in the NGC 2023 region.

Acknowledgements. We thank Christine Joblin and Laurent Verstraete for many fruitful discussions. ISO is an ESA project with instruments funded by ESA Member States and with the participation of ISAS and NASA.

\section{References}

Abergel, A., Miville-Deschênes, Désert, F. X., et al. 2000a, Exp. Astron., 10, 353

Abergel, A., André, P., Bacmann, A., et al. 2000b, in The Universe as Seen by ISO, ed. P. Cox, \& M. F. Kessler (ESA Pub., Noordwijk), SP 427, vol. 2, 615

Abergel, A., Bernard, J.-P., Boulanger, F., et al. 2002a, in Infrared and Submillimeter Space Astronomy: An International Colloquium to Honor the Memory of Guy Serra, EAS Publications Ser., vol. 4, in press

Abergel, A., Teyssier, D., Bernard, J. P., et al. 2002b, in prep. Allain, T., Leach, S., \& Sedlmayr, E. 1996, A\&A, 305, 602

Allamandola, L., Tielens, A. G. G. M., \& Barker, J. R. 1985, ApJ, 290, L25

Anthony-Twarog, B. J. 1982, AJ, 87, 1213

Bally, J., Langer, W. D., \& Liu, W. 1991, ApJ, 383, 645

Barnes, P. J., Crutcher, R. M., Richard, M., et al. 1989, ApJ, 342,883

Bernard, J.-P., Boulanger, F., \& Puget, J.-L. 1993, A\&A, 277, 609

Biviano, A., Sauvage, M., Gallais, P., et al. 1998, The ISOCAM Dark Current Calibration Report, available at http://www.iso.vilspa.esa.es/users/expl_lib/ CAM_list.html.

Blommaert, J. A. D. L., Boulanger, F., \& Okumura, K. 2001a, ISOCAM CVF photometry report, available at http://www.iso.vilspa.esa.es/users/expl_lib/ CAM_list.html.

Blommaert, J. A. D. L., Siebenmorgen, R., Coulais, A., et al. 2001b, The ISO handbook, vol. III (CAM - The ISO camera), version 1.2 , available at http://www.iso.vilspa.esa.es/manuals/HANDBOOK/III/ cam_hb

Borghesi, A., Bussoletti, E., \& Colangeli, L. 1987, ApJ, 314, 422

Boulanger, F., Falgarone, E., Puget, J.-L., et al. 1990, ApJ, 364,136

Boulanger, F., Abergel, A., Bernard, J.-P., et al. 1998a, in Star Formation with ISO, ed. J. L. Yun, \& R. Liseau (Astronomical Society of the Pacific, San Francisco), 15

Boulanger, F., Boisssel, P., Cesarsky, D., \& Ryter, C. 1998b, A\&A, 339, 194
Boulanger, F. 1999, in Solid Interstellar Matter: The ISO Revolution, ed. L. d'Hendecourt, C. Joblin, \& A. Jones (EDP Sciences and Springer-Verlag), 20

Cesarsky, C. J., Abergel, A., Agnèse, P., et al. 1996, A\&A, 315, L32

Cesarsky, D., Lequeux, J., Ryter, C., \& Gérin, M. 2000, A\&A, 354, L87

Chan, K.-W., Roellig, T. L., Onada, T., et al. 2001, ApJ, 546, 273

Cherchneff, I., Barker, J. R., \& Tielens, A. G. G. M. 1992, ApJ, 401, 269

Coulais, A., \& Abergel, A. 2000, A\&AS, 141, 533

Cox, P., Boulanger, F., Huggins, P. J., et al. 1998, ApJ, 495, 23

Dartois, E., \& D'Hendecourt, L. 1997, A\&A, 323, 534

Draine, B. T., \& Lee, H. M. 1984, ApJ, 285, 89

Gatley, I., Hasegawa, T., Suzuki, H., et al. 1987, ApJ, 318, L73

Greenberg, J. M., Gillette, J. S., Munoz Caro, G. M., et al. 2000, ApJ, 531, L71

Guillois, O., Nenner, I., Papoular, R., et al. 1996, ApJ, 464, 810

Habing, H. J. 1968, Bull. Astr. Netherlands, 19, 421

Hudgins, D., \& Allamamdola, L. J. 1995, J. Phys. Chem., 99, 3033

Hudgins, D. M., \& Sandford, S. A. 1998, J. Phys. Chem. A, 102, 329

Kelsall, T., Weiland, J. L., Franz, B. A., et al. 1998, ApJ, 508, 25

Jaffe, D. T., Zhou, S., Howe, J. E., et al. 1994, ApJ, 436, 203

Joblin, C., Boissel, P., \& Léger, A. 1995, A\&A, 299, 835

Joblin, C., Tielens, A. G. G. M., Geballe, T. R., et al. 1996, ApJ, 460, L119

Jones, A., \& d'Hendecourt, L. 2000, A\&A, 335, 1191

Kutner, M. L., Tucker, K. D., Chin, G., et al. 1977, ApJ, 215, 521

Langhoff, S. R. 1996, J. Phys. Chem., 100, 2819

Léger, A., \& Puget, J. L. 1984, A\&A, 137, L5

Leech, K., de Graauw, T., van den Ancker, M., et al. 2001, The ISO handbook, vol. VI (SWS), version 1.2, available at http://www.iso.vilspa.esa.es/manuals/HANDBOOK/VI/ sws_hb

Li, W., Evans, N. J. II, \& Lada, E. 1997, ApJ, 488, 277

Malin, D. 1987, Sky \& Telescope, 74, 253

Maddalena, R. J., Moscowitz, J., Thaddeus, P., et al. 1986, ApJ, 303, 375

Mattila, K., Lemke, D., Haikala, L. K., et al. 1996, A\&A, 315, L353

Miville-Deschênes, M.-A., Boulanger, F., Abergel, A., \& Bernard, J.-P. 2000, A\&AS, 147, 1

Miville-Deschênes, M.-A., Boulanger, F., Joncas, G., et al. 2002, A\&A, 381, 209

Moutou, C., Sellgren, K., Léger, A., et al. 1998, in Star Formation with ISO, ed. J. L. Yun, \& R. Liseau (Astronomical Society of the Pacific, San Francisco), 47

Moutou, C., Verstraete, L., Léger, A., Sellgren, K., \& Schmidt, W. 2000, A\&A, 354, 17

Nordh, L., Olofsson, G., Bontemps, S., et al. 1998, in Star Formation with ISO, ed. J. L. Yun, \& R. Liseau (Astronomical Society of the Pacific, San Francisco), 220

Panagia, N. 1973, AJ, 78, 929 
Papoular, R., Conard, J., Guiliano, M., et al. 1989, A\&A, 217, Tielens, A. G. G. M., Hony, S., van Kerckhoven, C., \& Peeters, 204

Perryman, M. A. C., Lindegren, L., Kovalevsky, J., et al. 1997, A\&A, 323, 49

Reipurth, B., \& Bouchet, P. 1984, A\&A, 137, L1 E. 2000, in The Universe as Seen by ISO, ed. P. Cox, \& M. F. Kessler (ESA Pub., Noordwijk), SP 427, vol. 2, 579 Uchida, K. I., Sellgren, K., Werner, M. W., et al. 2000, ApJ, 530,817

Sakata, A., Wada, S., Tanabe, T., \& Onaka, T. 1984, ApJ, 287, L51

van Kerckhoven, C., Hony, S., Peeters, E., et al. 2000, A\&A, 357, 1013

Schnaiter, M., Henning, Th., Mutschke, H., et al. 1999, ApJ, 519,687

Verstraete, L., Puget, J. L., Falgarone, E., et al. 1996, A\&A, 315, L337

Schutte, W. A., Tielens, A. G. G. M., \& Allamandola, L. J. 1993, ApJ, 415, 397

Verstraete, L., Pech, C., Moutou, C., et al. 2001, A\&A, 372, 981

Sellgren, K., Allamandola, L. J., Bregman, J. D, et al. 1985, ApJ, 299, 416

Sloan, G. C., Hayward, T. L., Allamandola, L. J., et al. 1999, ApJ, 513, L65

Szczepanski, J., Vala, M. 1993, ApJ, 414, 646

Weingartner, J. C., \& Draine, B. T. 2000, in The Universe as Seen by ISO, ed. P. Cox, \& M. F. Kessler (ESA Pub., Noordwijk), SP-427 II, 783

Weingartner, J. C., \& Draine, B. T. 2001, ApJ, 553, 581

Warren, W. H. Jr., \& Hesser, J. E. 1977, ApJS, 34, 115 